\title{
Plants are not humans
}

Advances in genomics have made it possible to use genome-wide association (GWA) studies to explore the genetic basis of common traits in humans, leading to advances in biological knowledge and technical expertise. The power of the GWA approach has now been extended successfully to Arabidopsis thaliana, in which results are proving to be strikingly different from those obtained in humans.

It is hoped that GWA studies in plants will shed light on the natural genetic variation that underlies adaptive and commercially important traits. Plant geneticists are aided in this task by the existence of inbred lines of $A$. thaliana, which can be phenotyped easily for many traits in different environments. Atwell et al. conducted their GWA study on almost 200 such lines by analysing associations between a genome-wide set of 250,000 SNPs and 107 traits across four categories: flowering, development, defence and element concentration.

The first striking finding was that, compared with humans, $A$. thaliana has a high degree of population structure (see also Further Reading and Websites below). This could potentially lead to a problematic number of spurious associations; however, the authors show that some of these false positives can be eliminated by using a mixed-model approach (which can correct for fine-scale structure). For example, previously known variants were easily identified - including those for disease resistance and for variation in sodium levels. However, association peaks were not always very sharp. In one case, a peak spanned $500 \mathrm{~kb}$ (encompassing 100 genes), showing that complex genetic architecture involving multiple correlated causative variants can be a further confounding factor - one that has previously not been recognized in humans.

Second, there is a higher probability than would be expected by chance that the SNPs that are strongly associated will include candidate SNPs (SNPs that are expected to be connected to a trait based on functional knowledge).

A third difference is that the number of samples used in the plant GWA study was very low; the study used a maximum of 192 lines, whereas most human GWA studies use thousands of individuals. This low sample size would seem to preclude identifying any association at all were it not for two additional advantages offered by $A$. thaliana: the existence of intermediate-frequency alleles of large effect (explaining up to $20 \%$ of the variation for several traits, compared with the preponderance of minor-effect alleles in humans) and the ability to control for environmental noise in the inbred lines. Both of these features 'amplify' the association signal, allowing true associations to stand out.

The GWA approach has some intrinsic limitations - for example, the ability only to identify alleles that are common. However, falling genotyping and sequencing costs and a growing list of new phenotypes means that such studies should have a bright future for characterizing the natural genetic variants that underlie quantitative traits in A. thaliana and other organisms.

Tanita Casci

ORIGINAL RESEARCH PAPER Atwell, S., Huang, Y. S., Vilhjálmsson, B. J. \& Willems, G. et al. Genome-wide association study of 107 phenotypes in Arabidopsis thaliana inbred lines. Nature 24 Mar 2010 (doi:10.1038/nature08800) FURTHER READING Platt, A. et al. The scale of population structure in Arabidopsis thaliana. PLoS Genet. 6, e1000843 (2010) WEBSITES

1001 Genomes Project: http://www.1001genomes.org Genome-wide association studies in Arabidopsis thaliana: http://arabidopsis.usc.edu

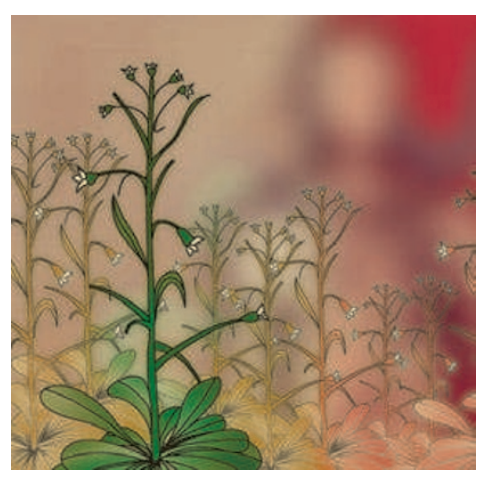

